\title{
碘催化下离子液体中 2-苯基-2-苯甲酰基喹唑啉衍生物的合成
}

\author{
张梅梅 $^{a} \quad$ 刘 蕴 ${ }^{b}$ 王香善*,a,b \\ ( ${ }^{a}$ 江苏省药用植物生物技术重点实验室 江苏师范大学 徐州 221116) \\ ( ${ }^{b}$ 江苏师范大学化学化工学院 徐州 221116)
}

\begin{abstract}
摘要 介绍了一种以离子液体为绿色介质合成 2,3-二氢化-2-苯基-2-苯甲酰基喹唑啉-4-酮衍生物的方法. 该方法是碘催 化下的 2-氨基苯甲酰胺和二苯基乙二酮反应, 产物 2,3-二氢化-2-苯基-2-苯甲酰基-3-苯乙基喹唑啉-4-酮(3f)的结构通过 $\mathrm{X}$ 射线衍射分析确证.

关键词＼cjkstart喹唑啉-4-酮; 2-氨基苯甲酰胺; 二苯基乙二䣳; 离子液体; 碘
\end{abstract}

\section{Synthesis of 2-Phenyl-2-benzoylquinazolin-4(1H)-one Derivatives in Ionic Liquids Catalyzed by lodine}

\author{
Zhang, Meimei ${ }^{a} \quad$ Liu, Yun ${ }^{b} \quad$ Wang, Xiangshan*,a,b \\ ( ${ }^{a}$ Key Laboratory of Biotechnology on Medical Plant of Jiangsu Province, Jiangsu Normal University, Xuzhou 221116) \\ ( ${ }^{b}$ School of Chemistry and Chemical Engineering, Jiangsu Normal University, Xuzhou 221116)
}

\begin{abstract}
A green method for the synthesis of 2,3-dihydro-2-benzoyl-2-phenylquinazolin-4(1H)-one derivatives, is described in high yields using ionic liquids as green media. The method involves a reaction of 2-aminobenzamide and benzil catalyzed by iodine. The structure of the product 2-benzoyl-3-phenethyl-2-phenyl-2,3-dihydroquinazolin-4(1H)-one (3f) was confirmed by X-ray diffraction analysis.
\end{abstract}

Keywords quinazolin-4(1H)-one; 2-aminobenzamide; benzil; ionic liquid; iodine

近年来，离子液体作为绿色化学的研究内容之一越 来越受到人们的重视. 因为离子液体本身既可以作为溶 剂，又可以作为反应催化剂，还具有不易燃，不易爆， 挥发性小和可以循环使用等优点，在有机合成中，作为 绿色反应介质在很多经典的反应中得到了广泛的应用, 主要包括氧化反应 ${ }^{[1]} 、$ 还原反应 ${ }^{[2]} 、$ Friedel-Crafts 反应 ${ }^{[3]}$ 、 Diels-Alder 反应 ${ }^{[4]}$ 和 Michael 加成反应等 ${ }^{[5]}$. 在杂环合成 中也已经得到很好的应用 ${ }^{[6]}$.

喹唑啉衍生物是一类重要的化合物, 具有诸多生物 和药理活性, 例如 2-取代的喹唑啉酮衍生物具有降压、 抗菌和抗癌等生物活性 ${ }^{[7]}$. 尽管已有很多文献报道过其 合成方法 ${ }^{[8]}$, 主要通过邻氨基苯甲酰胺和醛或酮的反 应 $^{[9]}$, 我们课题组 ${ }^{[10]}$ 已经成功将离子液体用作介质绿色 合成了结构多样化的喹唑啉衍生物, 本文报道离子液体 中将二苯基乙二酮作为羰基化合物, 与邻氨基苯甲酰胺
反应，合成系列的 2,3-二氢化-2-苯基-2-苯甲酰基喹唑 啉-4-酮衍生物.

\section{1 结果与讨论}

离子液体 $[\mathrm{BMIm}] \mathrm{Br}$ 中, 将邻氨基苯甲酰胺(1)和二 苯基乙二酮(2)在催化量的碘存在下, $80{ }^{\circ} \mathrm{C}$ 反应几小时, 可以较高产率得到相应的 2,3-二氢化-2-苯基-2-苯甲酰 基喹唑啉-4-酮衍生物 3 (Eq. 1).

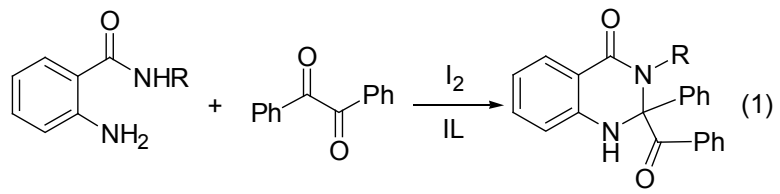

我们用 2-氨基- $N$-丙基苯甲酰胺(1a)和二苯基乙二 酮的反应为例, 对反应条件进行优化篮选(表 1). 在室温

\footnotetext{
*E-mail: xswang1974@yahoo.com

Received February 1, 2014; revised April 1, 2014; published online April 16, 2014.

Project supported by the National Natural Science Foundation of China (No. 20802061) and the College Industrialization Project of Jiangsu Province (No. JHB2012-31).

国家自然科学基金(No. 20802061)和江苏省高校产业化基金(No. JHB2013-31)资助项目.
} 
下和 $80{ }^{\circ} \mathrm{C}$ 反应通过 TLC 检测都没有发现产物 $\mathbf{3 a}$; 说明 不加入催化剂该反应不能进行. 加入催化剂 $5 \mathrm{~mol} \%$ 碘, 室温下只能监测到产物, $80{ }^{\circ} \mathrm{C}$ 下得到最高的产率 $(87 \%)$. 以不同的离子液体, 包括不同的咪唑阳离子和阴离子作 反应介质, 发现 $[\mathrm{BMIm}] \mathrm{Br}$ 对于这个反应的效果最好. 增加催化剂的用量到 10 和 $20 \mathrm{~mol} \%, 3 \mathrm{a}$ 的产量没有明显 的增高. 在常规的有机溶剂 THF 或 DMF 中的产率也相 对较低. 反应结束后, 向反应体系内加入少量的水析出 产物, 简单的过滤即可实现产物和离子液体分离. 减压 除去滤液中的水, $80{ }^{\circ} \mathrm{C}$ 真空干燥 $4 \mathrm{~h}$, 即可循环使用离 子液体, 上述的反应循环使用离子液体 4 次(表 2), 产物 3a 的产率还很高 $(85 \%)$.

表 1 不同条件下 $\mathbf{3 a}$ 的合成结果 ${ }^{a}$

Table 1 Synthetic results of 3a under different reaction conditions

\begin{tabular}{|c|c|c|c|c|}
\hline 序号 & 温度 $/{ }^{\circ} \mathrm{C}$ & $\mathrm{I}_{2} / \mathrm{mol} \%$ & 离子液体 ${ }^{b}$ & 产率 ${ }^{c} / \%$ \\
\hline 1 & r.t. & - & {$[\mathrm{BMIm}] \mathrm{Br}$} & 0 \\
\hline 2 & 80 & - & {$[\mathrm{BMIm}] \mathrm{Br}$} & 0 \\
\hline 3 & r.t. & 5 & {$[\mathrm{BMIm}] \mathrm{Br}$} & Trace \\
\hline 4 & 50 & 5 & {$[\mathrm{BMIm}] \mathrm{Br}$} & 58 \\
\hline 5 & 80 & 5 & {$[\mathrm{BMIm}] \mathrm{Br}$} & 87 \\
\hline 6 & 100 & 5 & {$[\mathrm{BMIm}] \mathrm{Br}$} & 87 \\
\hline 7 & 80 & 5 & [EMIm]Br & 84 \\
\hline 8 & 80 & 5 & [PMIm]Br & 85 \\
\hline 9 & 80 & 5 & {$[\mathrm{EMIm}]\left[\mathrm{BF}_{4}\right]$} & 80 \\
\hline 10 & 80 & 5 & {$[\mathrm{PMIm}]\left[\mathrm{BF}_{4}\right]$} & 83 \\
\hline 11 & 80 & 5 & {$[\mathrm{BMIm}]\left[\mathrm{BF}_{4}\right]$} & 83 \\
\hline 12 & 80 & 10 & {$[\mathrm{BMIm}] \mathrm{Br}$} & 83 \\
\hline 13 & 80 & 20 & {$[\mathrm{BMIm}] \mathrm{Br}$} & 85 \\
\hline 14 & Reflux & 5 & THF & 67 \\
\hline 15 & 80 & 5 & DMF & 79 \\
\hline
\end{tabular}

${ }^{a}$ 反应条件: $2 \mathrm{~mL}$ 溶剂, 1a $(0.178 \mathrm{~g}, 1.0 \mathrm{mmol})$ 和 $2(0.210 \mathrm{~g}, 1.05 \mathrm{mmol})$. ${ }^{b} \mathrm{BMIm}$ 为 1 -丁基-3-甲基咪唑阳离子; $\mathrm{EMIm}=1$-乙基-3-甲基咪唑阳离子; $\mathrm{PMIm}=1$-丙基-3-甲基咪唑阳离子. ${ }^{c}$ 分离产率.

表 2 循环使用离子液体 $3 \mathbf{a}$ 的合成结果 ${ }^{a}$

Table 2 Synthetic results of $\mathbf{3 a}$ in recycled ionic liquids

\begin{tabular}{ccccc}
\hline 次数 & 1 & 2 & 3 & 4 \\
\hline 产率 & 86 & 84 & 85 & 85 \\
\hline
\end{tabular}

根据篮选和优化的反应条件, 不同邻氨基苯甲酰胺 和 2 反应, 均得到相应的喹唑啉-4-酮产物(表 3). 所采用 的邻氨基苯甲酰胺中 $\mathrm{N}$-原子上的取代基, 无论是脂肪 族还是芳香族, 都没有很大的影响, 均可以得到很好的 产率. 通过 IR, ${ }^{1} \mathrm{H}$ NMR 和 HRMS 表征所有产物的结构, 数据均与产物 $\mathbf{3}$ 的结构相吻合; 产物 $\mathbf{3 f}$ 的结构还被 X 射线光衍射分析确证(图 1).

在晶体结构 $3 f$ 中, 喹唑啉结构中的嘧啶环采用半 椅式构象：原子 $\mathrm{N}(1), \mathrm{N}(2), \mathrm{C}(1), \mathrm{C}(2)$ 和 $\mathrm{C}(7)$ 共平面，原 子 $\mathrm{C}(8)$ 偏离这个平面为 0.553 (4) $\AA$; 这个平面与相连接 的苯环 $\mathrm{C}(16) \sim \mathrm{C}(21)$ 形成的二面角为 $68.1(1)^{\circ}$; 与相邻
表 3 离子液体中 $\mathbf{3 a} \sim 3$ 的合成结果

Table 3 Synthetic Results of $\mathbf{3 a} \sim \mathbf{3 1}$ in ionic liquids ${ }^{a}$

\begin{tabular}{clccc}
\hline 序号 & \multicolumn{1}{c}{$\mathrm{R}$} & 反应时间 $/ \mathrm{h}$ & 产物 & 产率 $^{b} / \%$ \\
\hline 1 & $n-\mathrm{Pr}$ & 8 & $\mathbf{3 a}$ & 87 \\
2 & $n-\mathrm{Bu}$ & 9 & $\mathbf{3 b}$ & 90 \\
3 & $\mathrm{PhCH}_{2}$ & 7 & $\mathbf{3 c}$ & 84 \\
4 & $4-\mathrm{MeC}_{6} \mathrm{H}_{4} \mathrm{CH}_{2}$ & 8 & $\mathbf{3 d}$ & 89 \\
5 & Furan-2-ylmethyl & 10 & $\mathbf{3 e}$ & 86 \\
6 & $\mathrm{Ph}\left(\mathrm{CH}_{2}\right)_{2}$ & 6 & $\mathbf{3 f}$ & 91 \\
7 & $4-\mathrm{MeOC}_{6} \mathrm{H}_{4}\left(\mathrm{CH}_{2}\right)_{2}$ & 7 & $\mathbf{3 g}$ & 90 \\
8 & $4-\mathrm{MeC}_{6} \mathrm{H}_{4}$ & 8 & $\mathbf{3 h}$ & 83 \\
9 & $4-\mathrm{FC}_{6} \mathrm{H}_{4}$ & 9 & $\mathbf{3 i}$ & 82 \\
10 & $4-\mathrm{MeOC}_{6} \mathrm{H}_{4}$ & 10 & $\mathbf{3 j}$ & 84 \\
11 & $4-i-\mathrm{PrC}_{6} \mathrm{H}_{4}$ & 9 & $\mathbf{3 k}$ & 86 \\
12 & $\mathrm{H}$ & 10 & $\mathbf{3 1}$ & 90 \\
\hline
\end{tabular}

反应条件: $2 \mathrm{~mL}$ [BMIm] Br, $1(1.0 \mathrm{mmol}), \mathbf{2}(0.210 \mathrm{~g}, 1.0 \mathrm{mmol})$ 和 $\mathrm{I}_{2}(13$ $\mathrm{mg}, 0.05 \mathrm{mmol}) .^{b}$ 分离产率.

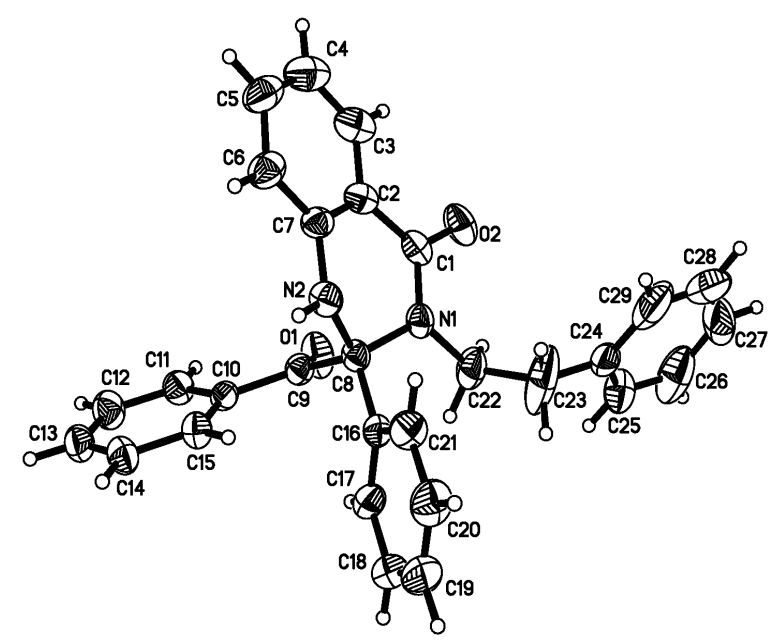

图 1 产物 $3 \mathrm{f}$ 的晶体结构图

Figure 1 The crystal structure of product $\mathbf{3 f}$

的苯环 $\mathrm{C}(2) \sim \mathrm{C}(7)$ 几乎平行, 形成的二面角为 4.1(2) ; 与苯甲酰基和苯乙基中的苯环形成的二面角分别为 70.(9) ${ }^{\circ}$ 和 $59.8(1)^{\circ}$.

根据产物的结构和文献报道 ${ }^{[10]}$, 我们认为碘是作 为 Lewis 酸，首先促进分子间羰基和氨基的缩合失水， 其次是催化分子内酰氨基和碳-氮双键的加成关环，具 体反应过程如 Eq. 2 所示.<smiles>[R]NC(=O)c1ccccc1N=C(C(=O)Oc1ccccc1)c1ccccc1N(CCO)c1ccccc1C(=O)N[R]</smiles>

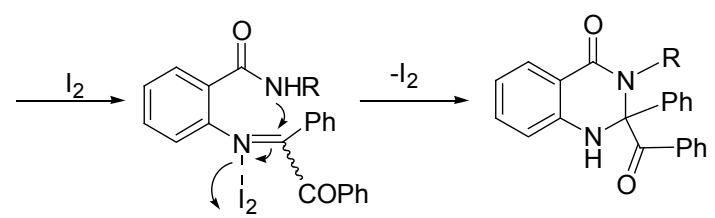




\section{2 结论}

介绍了一锅法合成 2,3-二氢化-2-苯基-2-苯甲酰基 喹唑啉-4-酮衍生物的方法, 具有产率高、反应条件温 和、分离简单和环境友好等优点. 并通过 IR, ${ }^{1} \mathrm{H}$ NMR 和 HRMS 表征其结构, 其中化合物 $\mathbf{3 f}$ 进一步通过 $\mathrm{X}$ 射 线衍射分析确证.

\section{3 实验部分}

\section{1 仪器与试剂}

熔点测定使用 XT-5 型数字熔点仪; 红外光谱采用 TENSOR 27 型红外光谱仪测定 $(\mathrm{KBr}$ 压片); 核磁共振氢 谱采用 Bruker AVANCE DPX-400 型核磁共振仪测定, DMSO- $d_{6}$ 为溶剂, TMS 为内标; 高分辨质谱在 Brukermicro-TOF-Q-MS 质谱仪上测定, $\mathrm{X}$ 射线衍射分析在 Bruker APEX-II 型四圆衍射仪上进行.

\section{2 实验方法}

干燥的 $50 \mathrm{~mL}$ 烧瓶中加入邻氨基苯甲酰胺(1) (1.0 $\mathrm{mmol}) 、$ 二苯基乙二酮 $(2)(0.210 \mathrm{~g}, 1.0 \mathrm{mmol}) 、$ 碘 $(13 \mathrm{mg}$, $0.05 \mathrm{mmol})$ 和离子液体 $[\mathrm{BMIm}] \mathrm{Br}(2 \mathrm{~mL})$. 摚拌加热到 $80{ }^{\circ} \mathrm{C}$ 保持 $6 \sim 10 \mathrm{~h}$, 冷却到室温加入 $5 \mathrm{~mL}$ 水, 过滤得到 粗品. 粗品用 $95 \% \mathrm{EtOH}$ 重结晶得到化合物 3.

2,3-二氢化-2-苯基-2-苯甲酰基-3-正丙基喹唑啉-4酮(3a): m.p. $217 \sim 218{ }^{\circ} \mathrm{C} ;{ }^{1} \mathrm{H}$ NMR (DMSO- $d_{6}, 400$ MHz) $\delta: 0.46\left(\mathrm{t}, J=7.2 \mathrm{~Hz}, 3 \mathrm{H}, \mathrm{CH}_{3}\right), 0.72 \sim 0.82(\mathrm{~m}, 1 \mathrm{H}$, $\mathrm{CH}), 0.98 \sim 1.07(\mathrm{~m}, 1 \mathrm{H}, \mathrm{CH}), 2.65 \sim 2.73(\mathrm{~m}, 1 \mathrm{H}, \mathrm{CH})$, $3.55 \sim 3.63(\mathrm{~m}, 1 \mathrm{H}, \mathrm{CH}), 6.64 \sim 6.66(\mathrm{~m}, 1 \mathrm{H}, \mathrm{ArH}), 6.68 \sim$ $6.72(\mathrm{~m}, 1 \mathrm{H}, \mathrm{ArH}), 7.13 \sim 7.18(\mathrm{~m}, 1 \mathrm{H}, \mathrm{ArH}), 7.33 \sim 7.37$ (m, 2H, ArH), $7.50 \sim 7.54(\mathrm{~m}, 4 \mathrm{H}, \operatorname{ArH}), 7.61 \sim 7.65(\mathrm{~m}$, $3 \mathrm{H}, \mathrm{ArH}), 7.67 \sim 7.70(\mathrm{~m}, 2 \mathrm{H}, \mathrm{ArH}), 8.02(\mathrm{~s}, 1 \mathrm{H}, \mathrm{NH})$; IR (KBr) v: 3312, 2983, 2960, 2934, 2873, 1684, 1627, 1585, $1507,1486,1465,1449,1439,1392,1361,1320,1262$, $1228,1184,1177,1155,1084,1067,1028,1007,938,865$, $808,761 \mathrm{~cm}^{-1}$. HRMS calcd for $\mathrm{C}_{24} \mathrm{H}_{22} \mathrm{~N}_{2} \mathrm{O}_{2} \mathrm{Na}[\mathrm{M}+\mathrm{Na}]^{+}$ 393.1579, found 393.1586.

2,3-二氢化-2-苯基-2-苯甲酰基-3-正丁基喹唑啉-4酩(3b): m.p. $210 \sim 212{ }^{\circ} \mathrm{C} ;{ }^{1} \mathrm{H}$ NMR (DMSO- $d_{6}, 400$ MHz) $\delta: 0.53\left(\mathrm{t}, J=7.2 \mathrm{~Hz}, 3 \mathrm{H}, \mathrm{CH}_{3}\right), 0.66 \sim 0.72(\mathrm{~m}, 1 \mathrm{H}$, $\mathrm{CH}), 0.81 \sim 0.95\left(\mathrm{~m}, 2 \mathrm{H}, \mathrm{CH}_{2}\right), 1.01 \sim 1.03(\mathrm{~m}, 1 \mathrm{H}, \mathrm{CH})$, $2.67 \sim 2.76(\mathrm{~m}, 1 \mathrm{H}, \mathrm{CH}), 3.55 \sim 3.65(\mathrm{~m}, 1 \mathrm{H}, \mathrm{CH}), 6.64(\mathrm{~d}$, $J=8.0 \mathrm{~Hz}, 1 \mathrm{H}, \mathrm{ArH}), 6.67 \sim 6.72(\mathrm{~m}, 1 \mathrm{H}, \mathrm{ArH}), 7.13 \sim$ $7.18(\mathrm{~m}, 1 \mathrm{H}, \mathrm{ArH}), 7.33 \sim 7.37(\mathrm{~m}, 2 \mathrm{H}, \mathrm{ArH}), 7.50 \sim 7.55$ (m, 4H, ArH), 7.61 7.65 (m, 3H, ArH), 7.67 7.69 (m, 2H, ArH), 8.02 (s, 1H, NH); IR (KBr) v: 3298, 2962, 2940, $2875,1687,1627,1585,1507,1486,1450,1395,1362$,
1324, 1313, 1228, 1180, 1168, 1154, 1070, 866, 814, 761 $\mathrm{cm}^{-1}$. HRMS calcd for $\mathrm{C}_{25} \mathrm{H}_{24} \mathrm{~N}_{2} \mathrm{O}_{2} \mathrm{Na}[\mathrm{M}+\mathrm{Na}]^{+}$ 407.1735, found 407.1750.

2,3-二氢化-2-苯基-2-苯甲酰基-3-芐基喹唑啉-4-酮 (3c): m.p. $210 \sim 212{ }^{\circ} \mathrm{C} ;{ }^{1} \mathrm{H}$ NMR (DMSO- $d_{6}, 400 \mathrm{MHz}$ ) $\delta: 3.76(\mathrm{~d}, J=16.0 \mathrm{~Hz}, 1 \mathrm{H}, \mathrm{CH}), 5.25(\mathrm{~d}, J=16.0 \mathrm{~Hz}, 1 \mathrm{H}$, $\mathrm{CH}), 6.67 \sim 6.69(\mathrm{~m}, 1 \mathrm{H}, \mathrm{ArH}), 6.70 \sim 6.75(\mathrm{~m}, 3 \mathrm{H}, \mathrm{ArH})$, $7.01 \sim 7.04(\mathrm{~m}, 3 \mathrm{H}, \mathrm{ArH}), 7.17 \sim 7.19(\mathrm{~m}, 1 \mathrm{H}, \operatorname{ArH})$, $7.27 \sim 7.31(\mathrm{~m}, 2 \mathrm{H}, \operatorname{ArH}), 7.32 \sim 7.39(\mathrm{~m}, 3 \mathrm{H}, \operatorname{ArH})$, $7.45 \sim 7.49(\mathrm{~m}, 2 \mathrm{H}, \operatorname{ArH}), 7.51 \sim 7.53(\mathrm{~m}, 1 \mathrm{H}, \operatorname{ArH})$, $7.65 \sim 7.67(\mathrm{~m}, 2 \mathrm{H}, \mathrm{ArH}), 7.68 \sim 7.71(\mathrm{~m}, 1 \mathrm{H}, \mathrm{ArH}), 8.12$ (s, 1H, NH); IR (KBr) v: 3324, 3300, 3059, 3031, 2940, 1683, 1625, 1586, 1508, 1486, 1448, 1431, 1382, 1351, 1321, 1260, 1226, 1181, 1168, 1153, 968, 877, 866, 735 $\mathrm{cm}^{-1}$. HRMS calcd for $\mathrm{C}_{28} \mathrm{H}_{22} \mathrm{~N}_{2} \mathrm{O}_{2} \mathrm{Na}[\mathrm{M}+\mathrm{Na}]^{+}$ 441.1579 , found 441.1582 .

2,3-二氢化-2-苯基-2-苯甲酰基-3-(4-甲基芐基)喹唑 啉-4-酮(3d): m.p. $188 \sim 189{ }^{\circ} \mathrm{C} ;{ }^{1} \mathrm{H}$ NMR (DMSO- $d_{6}, 400$ MHz) $\delta: 2.16\left(\mathrm{~s}, 3 \mathrm{H}, \mathrm{CH}_{3}\right), 3.73(\mathrm{~d}, J=16.0 \mathrm{~Hz}, 1 \mathrm{H}, \mathrm{CH})$, $5.18(\mathrm{~d}, J=16.0 \mathrm{~Hz}, 1 \mathrm{H}, \mathrm{CH}), 6.60(\mathrm{~d}, J=7.6 \mathrm{~Hz}, 2 \mathrm{H}$, ArH), $6.67(\mathrm{~d}, J=8.0 \mathrm{~Hz}, 1 \mathrm{H}, \mathrm{ArH}), 6.71 \sim 6.74(\mathrm{~m}, 1 \mathrm{H}$, $\operatorname{ArH}), 6.84(\mathrm{~d}, J=8.0 \mathrm{~Hz}, 2 \mathrm{H}, \mathrm{ArH}), 7.16 \sim 7.20(\mathrm{~m}, 1 \mathrm{H}$, ArH), $7.30 \sim 7.35(\mathrm{~m}, 4 \mathrm{H}, \mathrm{ArH}), 7.38 \sim 7.42(\mathrm{~m}, 1 \mathrm{H}, \mathrm{ArH})$, $7.48 \sim 7.53$ (m, 3H, ArH), $7.64 \sim 7.69$ (m, 3H, ArH), 8.11 (s, $1 \mathrm{H}, \mathrm{NH}$ ); ${ }^{13} \mathrm{C}$ NMR (DMSO- $\left.d_{6}, 100 \mathrm{MHz}\right) \delta: 20.5$, 46.8, 83.7, 114.2, 114.6, 118.1, 126.4, 127.7, 128.0, 128.2, $128.3,129.2,129.7,130.0,132.9,133.3,133.6,134.8$, 135.79, 135.83, 144.5, 164.2, 198.0; IR (KBr) v: 3287, 3060, 3026, 2925, 1687, 1631, 1616, 1579, 1510, 1487, $1448,1430,1385,1347,1319,1301,1265,1225,1182$, $1157,1119,967,890,813,759 \mathrm{~cm}^{-1}$. HRMS calcd for $\mathrm{C}_{29} \mathrm{H}_{24} \mathrm{~N}_{2} \mathrm{O}_{2} \mathrm{Na}[\mathrm{M}+\mathrm{Na}]^{+}$455.1735, found 455.1740.

2,3-二氢化-2-苯基-2-苯甲酰基-3-(2-㫙喃甲基)喹唑 啉-4-酮(3e): m.p. $244 \sim 246{ }^{\circ} \mathrm{C} ;{ }^{1} \mathrm{H}$ NMR (DMSO- $d_{6}, 400$ MHz) $\delta: 3.84(\mathrm{~d}, J=16.4 \mathrm{~Hz}, 1 \mathrm{H}, \mathrm{CH}), 5.07$ (d, $J=16.4$ $\mathrm{Hz}, 1 \mathrm{H}, \mathrm{CH}), 5.48 \sim 5.49(\mathrm{~m}, 1 \mathrm{H}, \mathrm{ArH}), 6.11 \sim 6.12(\mathrm{~m}$, $1 \mathrm{H}, \operatorname{ArH}), 6.66(\mathrm{~d}, J=8.0 \mathrm{~Hz}, 1 \mathrm{H}, \operatorname{ArH}), 6.70 \sim 6.74(\mathrm{~m}$, $1 \mathrm{H}, \mathrm{ArH}), 7.16 \sim 7.20(\mathrm{~m}, 1 \mathrm{H}, \mathrm{ArH}), 7.26 \sim 7.27(\mathrm{~m}, 1 \mathrm{H}$, $\mathrm{ArH}), 7.32 \sim 7.36(\mathrm{~m}, 2 \mathrm{H}, \mathrm{ArH}), 7.40 \sim 7.44(\mathrm{~m}, 2 \mathrm{H}, \mathrm{ArH})$, $7.46 \sim 7.50(\mathrm{~m}, 1 \mathrm{H}, \operatorname{ArH}), 7.52 \sim 7.55(\mathrm{~m}, 3 \mathrm{H}, \operatorname{ArH})$, $7.65 \sim 7.68$ (m, 3H, ArH), $8.12(\mathrm{~s}, 1 \mathrm{H}, \mathrm{NH})$; IR $(\mathrm{KBr}) v$ : 3304, 3095, 3057, 3038, 2933, 1678, 1632, 1509, 1501, $1486,1448,1420,1389,1375,1328,1276,1226,1183$, $1167,1145,1118,1073,1029,1009,953,928,886,871$, $806,759 \mathrm{~cm}^{-1}$. HRMS calcd for $\mathrm{C}_{26} \mathrm{H}_{20} \mathrm{~N}_{2} \mathrm{O}_{3} \mathrm{Na}[\mathrm{M}+\mathrm{Na}]^{+}$ 
431.1372, found 431.1369 .

2,3-二氢化-2-苯基-2-苯甲酰基-3-苯乙基喹唑啉-4酥(3f): m.p. $178 \sim 179{ }^{\circ} \mathrm{C} ;{ }^{1} \mathrm{H}$ NMR (DMSO- $d_{6}, 400$ $\mathrm{MHz}) \delta: 1.93 \sim 2.00(\mathrm{~m}, 1 \mathrm{H}, \mathrm{CH}), 2.50 \sim 2.55(\mathrm{~m}, 1 \mathrm{H}$, $\mathrm{CH}), 2.82 \sim 2.88(\mathrm{~m}, 1 \mathrm{H}, \mathrm{CH}), 3.72 \sim 3.79(\mathrm{~m}, 1 \mathrm{H}, \mathrm{CH})$, $6.66 \sim 6.74(\mathrm{~m}, 4 \mathrm{H}, \mathrm{ArH}), 7.09 \sim 7.13(\mathrm{~m}, 1 \mathrm{H}, \mathrm{ArH})$, $7.15 \sim 7.20(\mathrm{~m}, 3 \mathrm{H}, \mathrm{ArH}), 7.34 \sim 7.38(\mathrm{~m}, 2 \mathrm{H}, \mathrm{ArH})$, $7.51 \sim 7.55(\mathrm{~m}, 1 \mathrm{H}, \mathrm{ArH}), 7.57 \sim 7.65(\mathrm{~m}, 3 \mathrm{H}, \mathrm{ArH})$, $7.66 \sim 7.71(\mathrm{~m}, 5 \mathrm{H}, \mathrm{ArH}), 8.10(\mathrm{~s}, 1 \mathrm{H}, \mathrm{NH})$; IR (KBr) $v$ : 3311, 3059, 3026, 2942, 1686, 1636, 1615, 1578, 1509, $1488,1449,1435,1385,1355,1333,1315,1277,1225$, $1180,1156,1119,812,753,718 \mathrm{~cm}^{-1}$. HRMS calcd for $\mathrm{C}_{29} \mathrm{H}_{24} \mathrm{~N}_{2} \mathrm{O}_{2} \mathrm{Na}[\mathrm{M}+\mathrm{Na}]^{+} 455.1735$, found 455.1744 .

2,3-二氢化-2-苯基-2-苯甲酰基-3-(4-甲氧基苯乙基) 喹唑啉 -4- 酤 (3g): m.p. $206 \sim 208{ }^{\circ} \mathrm{C} ;{ }^{1} \mathrm{H}$ NMR $\left(\mathrm{DMSO}-d_{6}, 400 \mathrm{MHz}\right) \delta: 1.86 \sim 1.94(\mathrm{~m}, 1 \mathrm{H}, \mathrm{CH}), 2.41 \sim$ $2.46(\mathrm{~m}, 1 \mathrm{H}, \mathrm{CH}), 2.78 \sim 2.86(\mathrm{~m}, 1 \mathrm{H}, \mathrm{CH}), 3.67 \sim 3.76$ $\left(\mathrm{m}, 4 \mathrm{H}, \mathrm{CH}+\mathrm{CH}_{3} \mathrm{O}\right), 6.59(\mathrm{~d}, J=8.4 \mathrm{~Hz}, 2 \mathrm{H}, \mathrm{ArH}), 6.66$ $(\mathrm{d}, J=8.0 \mathrm{~Hz}, 1 \mathrm{H}, \mathrm{ArH}), 6.70 \sim 6.74(\mathrm{~m}, 3 \mathrm{H}, \mathrm{ArH}), 7.16 \sim$ $7.20(\mathrm{~m}, 1 \mathrm{H}, \mathrm{ArH}), 7.36(\mathrm{t}, J=7.6 \mathrm{~Hz}, 2 \mathrm{H}, \mathrm{ArH}), 7.51 \sim$ 7.71 (m, 9H, ArH), 8.09 (s, 1H, NH); IR (KBr) v: 3345, 3060, 3030, 2951, 2935, 2913, 1686, 1634, 1615, 1505, 1487, 1447, 1386, 1360, 1324, 1310, 1248, 1223, 1178, 1120, 1031, 752, 717, $694 \mathrm{~cm}^{-1}$. HRMS calcd for $\mathrm{C}_{30} \mathrm{H}_{26} \mathrm{~N}_{2} \mathrm{O}_{3} \mathrm{Na}[\mathrm{M}+\mathrm{Na}]^{+}$485.1841, found 485.1858 .

2,3-二氢化-2-苯基-2-苯甲酰基-3-(4-甲基苯基)喹唑 啉-4-酩(3h): m.p. 228 230 ${ }^{\circ} \mathrm{C} ;{ }^{1} \mathrm{H}$ NMR (DMSO- $d_{6}, 400$ $\mathrm{MHz}) \delta: 2.15\left(\mathrm{~s}, 3 \mathrm{H}, \mathrm{CH}_{3}\right), 6.74 \sim 6.89(\mathrm{~m}, 5 \mathrm{H}, \mathrm{ArH})$, $7.21 \sim 7.33(\mathrm{~m}, 9 \mathrm{H}, \mathrm{ArH}), 7.46 \sim 7.50(\mathrm{~m}, 1 \mathrm{H}, \mathrm{ArH})$, $7.61 \sim 7.64(\mathrm{~m}, 2 \mathrm{H}, \mathrm{ArH}), 7.67 \sim 7.70(\mathrm{~m}, 1 \mathrm{H}, \mathrm{ArH}), 8.25$ (s, 1H, NH); IR (KBr) v: 3270, 3059, 3034, 3001, 1682, $1630,1609,1579,1513,1485,1449,1371,1339,1227$, $1179,1157,1107,1025,884,841,804,755 \mathrm{~cm}^{-1}$. HRMS calcd for $\mathrm{C}_{28} \mathrm{H}_{23} \mathrm{~N}_{2} \mathrm{O}_{2}[\mathrm{M}+\mathrm{H}]^{+}$419.1760, found 419.1773 .

2,3-二氢化-2-苯基-2-苯甲酰基-3-(4-氟苯基)喹唑啉4-酮(3i): m.p. $228 \sim 230{ }^{\circ} \mathrm{C} ;{ }^{1} \mathrm{H}$ NMR (DMSO- $d_{6}, 400$ $\mathrm{MHz}) \delta: 6.75 \sim 7.01(\mathrm{~m}, 5 \mathrm{H}, \mathrm{ArH}), 7.23 \sim 7.34(\mathrm{~m}, 9 \mathrm{H}$, ArH), $7.48 \sim 7.51(\mathrm{~m}, 1 \mathrm{H}, \operatorname{ArH}), 7.63$ (d, J=7.6 Hz, 2H, ArH), 7.69 (d, $J=8.0 \mathrm{~Hz}, 1 \mathrm{H}, \mathrm{ArH}), 8.33$ (s, 1H, NH); IR (KBr) $v: 3328,3071,3035,1681,1638,1615,1575,1506$, 1484, 1446, 1364, 1272, 1222, 1186, 1151, 1091, 1017, $1003,842,813,751 \mathrm{~cm}^{-1}$. HRMS calcd for $\mathrm{C}_{27} \mathrm{H}_{20} \mathrm{FN}_{2} \mathrm{O}_{2}$ $[\mathrm{M}+\mathrm{H}]^{+}$421.1345, found 421.1333 .

2,3-二氢化-2-苯基-2-苯甲酰基-3-(4-甲氧基苯基)喹 唑啉-4-酮(3j): m.p. 222 $223{ }^{\circ} \mathrm{C} ;{ }^{1} \mathrm{H}$ NMR (DMSO- $d_{6}$, $400 \mathrm{MHz}) \delta: 3.63\left(\mathrm{~s}, 3 \mathrm{H}, \mathrm{OCH}_{3}\right), 6.58 \sim 6.78(\mathrm{~m}, 5 \mathrm{H}$, ArH), $7.21 \sim 7.33(\mathrm{~m}, 9 \mathrm{H}, \mathrm{ArH}), 7.46 \sim 7.50(\mathrm{~m}, 1 \mathrm{H}, \mathrm{ArH})$, $7.62(\mathrm{~d}, J=7.6 \mathrm{~Hz}, 2 \mathrm{H}, \mathrm{ArH}), 7.68(\mathrm{~d}, J=7.6 \mathrm{~Hz}, 1 \mathrm{H}$, ArH), 8.26 (s, 1H, NH); IR (KBr) v: 3262, 3059, 3002, 2934, 2837, 1697, 1634, 1611, 1582, 1511, 1485, 1450, 1368, 1295, 1247, 1227, 1182, 1157, 1105, 1030, 833, 813, $755 \mathrm{~cm}^{-1}$. HRMS calcd for $\mathrm{C}_{28} \mathrm{H}_{22} \mathrm{~N}_{2} \mathrm{O}_{3} \mathrm{Na}[\mathrm{M}+\mathrm{Na}]^{+}$ 457.1528, found 457.1526.

2,3-二氢化-2-苯基-2-苯甲酰基-3-(4-异丙基苯基)喹 唑啉-4-酩(3k): m.p. $210 \sim 213{ }^{\circ} \mathrm{C} ;{ }^{1} \mathrm{H}$ NMR (DMSO- $d_{6}$, $400 \mathrm{MHz}) \delta: 1.07\left(\mathrm{dd}, J=6.8,2.4 \mathrm{~Hz}, 6 \mathrm{H}, 2 \mathrm{CH}_{3}\right), 2.68 \sim$ $2.76(\mathrm{~m}, 1 \mathrm{H}, \mathrm{CH}), 6.74 \sim 6.78(\mathrm{~m}, 2 \mathrm{H}, \mathrm{ArH}), 6.93 \sim 6.95$ (m, 3H, ArH), $7.21 \sim 7.32$ (m, 9H, ArH), 7.47 (d, J=7.6 $\mathrm{Hz}, 1 \mathrm{H}, \mathrm{ArH}), 7.63$ (d, $J=7.6 \mathrm{~Hz}, 2 \mathrm{H}, \mathrm{ArH}), 7.69$ (d, $J=$ $7.6 \mathrm{~Hz}, 1 \mathrm{H}, \mathrm{ArH}), 8.29$ (s, 1H, NH); IR (KBr) v: 3300, 3061, 3037, 2962, 2924, 1696, 1649, 1613, 1580, 1499, $1485,1449,1417,1360,1270,1233,1181,1160,1100$, 1083, 1050, 1026, 1006, 966, 884, 845, 833, 812, 757 $\mathrm{cm}^{-1}$. HRMS calcd for $\mathrm{C}_{30} \mathrm{H}_{26} \mathrm{~N}_{2} \mathrm{O}_{2} \mathrm{Na}[\mathrm{M}+\mathrm{Na}]^{+}$ 469.1892, found 469.1906.

2,3-二氢化-2-苯基-2-苯甲酰基喹唑啉-4-酤 (3I): m.p. $237 \sim 238{ }^{\circ} \mathrm{C} ;{ }^{1} \mathrm{H}$ NMR (DMSO- $\left.d_{6}, 400 \mathrm{MHz}\right) \delta$ : $6.68 \sim 6.72(\mathrm{~m}, 1 \mathrm{H}, \mathrm{ArH}), 6.87(\mathrm{~d}, J=8.0 \mathrm{~Hz}, 1 \mathrm{H}, \mathrm{ArH})$, $7.25 \sim 7.29(\mathrm{~m}, 1 \mathrm{H}, \mathrm{ArH}), 7.38 \sim 7.48(\mathrm{~m}, 5 \mathrm{H}, \mathrm{ArH})$, $7.53 \sim 7.59(\mathrm{~m}, 3 \mathrm{H}, \mathrm{ArH}), 7.70 \sim 7.74(\mathrm{~m}, 3 \mathrm{H}, \mathrm{ArH}), 8.94$ (s, 1H, NH), 9.10 (s, 1H, NH); IR (KBr) v: 3375, 3158, 3082, 1675, 1650, 1616, 1569, 1514, 1498, 1486, 1448, 1370, 1229, 1167, 1084, 1034, 826, 795, 742, 715, 703, $693 \mathrm{~cm}^{-1}$. HRMS calcd for $\mathrm{C}_{21} \mathrm{H}_{17} \mathrm{~N}_{2} \mathrm{O}_{2}[\mathrm{M}+\mathrm{H}]^{+}$ 329.1290 , found 329.1316 .

辅助材料(Supporting Information) 化合物 3a $\sim 31$ 的 氢谱和化合物 3d 的碳谱图. 这些材料可以免费从本刊 网站(http://sioc-journal.cn/)上下载.

\section{References}

[1] Howarth, J. Tetrahedron Lett. 2000, 41, 6627.

[2] Howarth, J.; James, P.; Dai, J. F. Tetrahedron Lett. 2001, 42, 7517.

[3] Khadilkar, B. M.; Rebeiro, G. L. Synth. Commun. 2000, 30, 1605.

[4] Lee, C. W. Tetrahedron Lett. 1999, 40, 2461.

[5] (a) DellcAnna, M. M.; Gallo, V.; Mastrorilli, P.; Nobile, C. F.; Romanazzi, G.; Suranna, G. P. Chem. Commun. 2002, 434. (b) Yi, F. P.; Zhang, X.; Sun, H. Y.; Chen, S. H. Acta Chim. Sinica 2012, 70, 741 (in Chinese).

(易封萍, 张旋, 孙海洋, 陈世洪, 化学学报, 2012, 70, 741.)

(c) Song, J.; Zhang, B.; Shi, J.; Ma, J.; Yang, G.; Han, B. Chin. J. Chem. 2012, 30, 2079. 
[6] (a) Dupont, J.; de Souza, R. F.; Suarez, P. A. Z. Chem. Rev. 2002, $102,3667$.

(b) Martins, M. A. P.; Frizzo, C. P.; Moreira, D. N.; Zanatta, N.; Bonacorso, H. G. Chem. Rev. 2008, 108, 2015.

(c) Welton, T. Chem. Rev. 1999, 99, 2071.

[7] (a) Alagarsamy, V.; Pathak, U. S. Bioorg. Med. Chem. 2007, 15, 345.

(b) Murugan, V.; Kulkarni, M.; Anand, R. M.; Kumar, E. P.; Suresh, B.; Reddy, V. M. Asian J. Chem., 2006, 18, 900.

(c) Selvam, P.; Girija, K.; Nagarajan, G.; Clerco, D. E. Indian J. Pharm. Sci. 2005, 67, 484.

[8] (a) Liu, C. E.; Yu, Q. Y.; Tang, J. H.; Li, J. R. Chin. J. Org. Chem. 2012, 32, 532 (in Chinese).

(刘长娥, 于琪瑶, 唐健红, 李加荣, 有机化学, 2012, 32, 532.)

(b) Shi, D. Q.; Dou, G. L.; Zhou, Y. Synthesis 2008, 2000.

(c) Wang, S. L.; Sheng, J.; Tu, S. J.; Wang, X. S. Chin. J. Org. Chem. 2011, 31, 1522 (in Chinese).

(王树良, 盛洁, 屠树江, 王香善, 有机化学, 2011, 31, 1522.)

(d) Fu, H. Chin. J. Org. Chem. 2009, 29, 488 (in Chinese).

(付华, 有机化学, 2009, 29, 488.)

(e) Molina, P.; Alajarin, M.; Vidal, A. Tetrahedron Lett. 1988, 29,
3849.

(f) Akazome, M.; Kondo, T.; Watanabe, Y. J. Org. Chem. 1993, 58, 310 .

(g) Dou, G. L.; Wang, M. M.; Shi, D. Q. J. Comb. Chem. 2009, 11, 151.

(h) Dou. G. L.; Shi, D. Q.; Li, Y. H. J. Comb. Chem. 2010, 12, 195. (i) Wang, M. M.; Dou, G. L.; Shi, D. Q. J. Comb. Chem. 2010, 12, 582 .

(j) Hu, Y.; Wang, M. M.; Chen, H.; Shi, D. Q. Tetrahedron 2011, 67, 9342.

[9] (a) Shaabani, A.; Maleki, A.; Mofakham, H. Synth. Commun. 2008, 38,3751 .

(b) Sharma, S. D.; Verinder, K. Synthesis 1989, 677.

(c) Reddy, P. S. N.; Reddy, P. P. Indian J. Chem. B 1988, 27, 135.

(d) Wang, S. L.; Yang, K.; Wang, X. S. Chin. J. Org. Chem. 2011, 31, 1235 (in Chinese).

(王树良, 杨科, 王香善, 有机化学, 2011, 31, 1235.)

[10] (a) Wang, X. S.; Yang, K.; Zhou, J.; Tu, S. J. J. Comb. Chem. 2010, 12,417

(b) Wang, X. S.; Sheng, J.; Yang, K.; Li, Y. L. J. Comb. Chem. 2011, 13, 196.

(Li, L.; Lu, Z.) 